\title{
Coulomb Interactions in a Focused Ion Beam System with a Dynamic Corrected Deflection Field
}

\author{
Wenping $\mathrm{Li}^{1, \mathrm{a}^{*}}$, Qian $\mathrm{Li}^{1, \mathrm{~b}}$ and Junbiao $\mathrm{Liu}^{2, \mathrm{c}}$ \\ ${ }^{1}$ School of Physics and Nuclear Energy Engineering, Beihang University, Beijing, China \\ ${ }^{2}$ Institute of Electrical Engineering, Chinese Academy of Sciences, Beijing, China \\ aliwp@buaa.edu, bliqian1314@buaa.edu, ${ }^{\mathrm{c} l i u j b @ m a i l . i e e . a c . c n ~}$
}

Keywords: Coulomb interactions; dynamic correction; three-dimension ion trajectory

Abstract. Focused ion beam systems complement conventional processing methods and can be used to prototype and modify a diverse range of nano-devices and sensors. Controllable incident focused ion beam is difficult to obtain due to Coulomb interactions among ions. In this paper, Coulomb interactions of a practical FIB system with a dynamic corrected deflection field are obtained through solving Newton-Lorentz equations in three-dimension (3D). Firstly, the principle of the dynamic correction is analyzed and its 3D realization is given. Secondly, the principle of N-body Monte Carlo method is researched in detail before the source parameter, the 3D field, and the number of $\mathrm{N}$ are determined. In the end, the equations are solved by fifth-order Runge-Kutta algorithm and 3D ion trajectory is given for a 30kV FIB through the Mebs software. Simulation results coincide to the experimental those from our Canion $31^{+}$system.

\section{Introduction}

Focused ion beam and its relative systems are versatile tools for nanoengineering and nanoscience applications. These systems complement conventional processing methods and can be used to prototype and modify a diverse range of nano-devices and sensors [1-3]. The quality and precision of FIB fabrication mainly depend on parameters of incident focused ion beam such as velocity, the spot, and the beam current density distribution. As ion is much heavier than electron, Coulomb ion-ion interactions play an important role in the spot and beam current density [1-2]. Much attention was paid to Coulomb interactions in the Ga LMIS of FIB systems [1-3], as the current density at the tip reaches above $10^{12} \mathrm{Am}^{-2}$. P. Kruit paid more attention to Coulomb interactions of two-lens FIB column under different imaging modes [4-5]. However, error is introduced as they have ignored the deflecting field which is essential to a real FIB system. In this paper, Coulomb interactions of a practical FIB system with a dynamic corrected deflection field are obtained through solving 3D Newton-Lorentz equations. 3D ion trajectory is given and the simulation results are analyzed with some experiments on our FIB-SEM dual-beam system.

\section{Three-dimension Dynamic Correction Field}

According to the dynamic correction theory [6], the deflection field curvature can be corrected by a dynamic focus lens and the deflection astigmatism by an octupole stigmator

\section{Principle of Dynamic Correction}

The dynamic focus lens has the same field distribution as the focusing lens to produce a negative behavior, whose polar coordinates form can be expressed as

$$
\phi(z, r)=\phi(z)-\frac{1}{4} \phi^{\prime \prime}(z) r^{2}+\frac{1}{64} \phi^{(4)}(z) r^{4}+\mathrm{L} .
$$

The field distribution of an octupole stigmator can be decomposed into two quadrupole fields as given in Eq. (2) along the XOY and POQ planes as shown in Fig. 1.

$$
\phi_{a}(r, \theta, z)=V_{a} \phi_{2}(r, z) \cos 2 \theta-V_{a} \phi_{6}(r, z) \cos 6 \theta+\mathrm{L}
$$


$\phi_{b}(r, \theta, z)=V_{b} \phi_{2}(r, z) \sin 2 \theta-V_{b} \phi_{6}(r, z) \sin 6 \theta+\mathrm{L}$

where $(r, \theta, z)$ is the polar coordinates, $\phi_{2}(r, z)$ and $\phi_{6}(r, z)$ are the second and sixth harmonic components on the $(r, z)$ plane.

Considering the superposition of electric signals, the objective lens can also work as a dynamic focus lens if we apply the suitable excitation as shown in Fig.2 and the two pre-lens deflectors can as two stigmators in Fig.3. Deflection field curvature, deflection distortion and astigmatism will be corrected by applying suitable potentials on the objective lens and the deflectors [7].

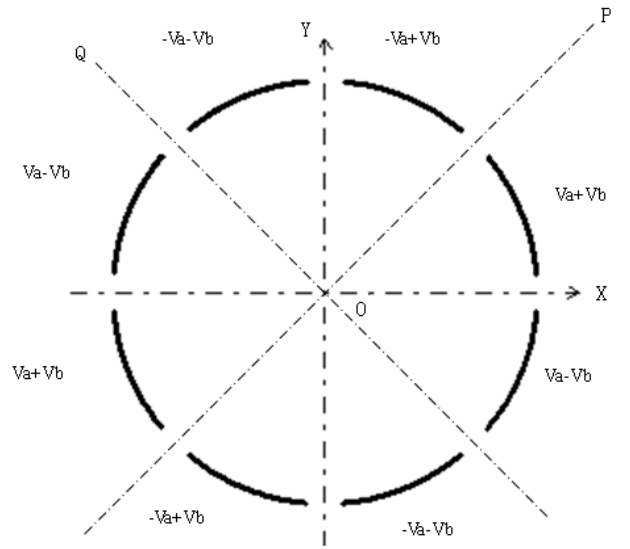

Fig.1 Schematic diagram of the stigmator

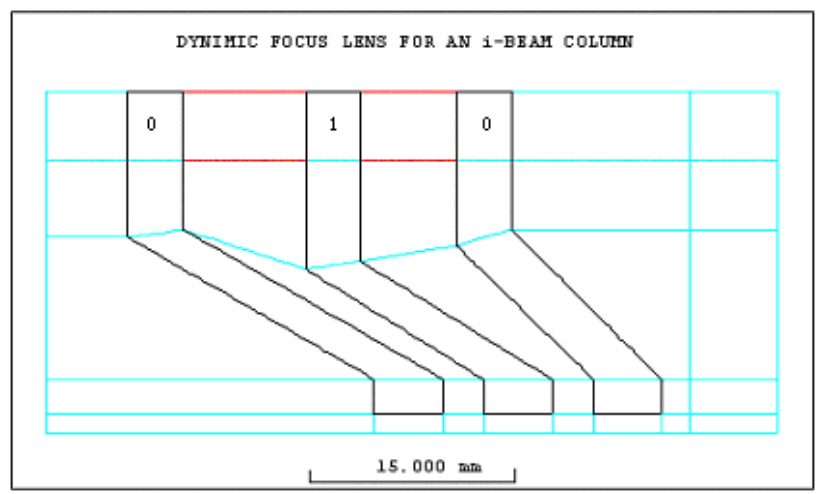

Fig.2 Equivalent dynamic focus lens
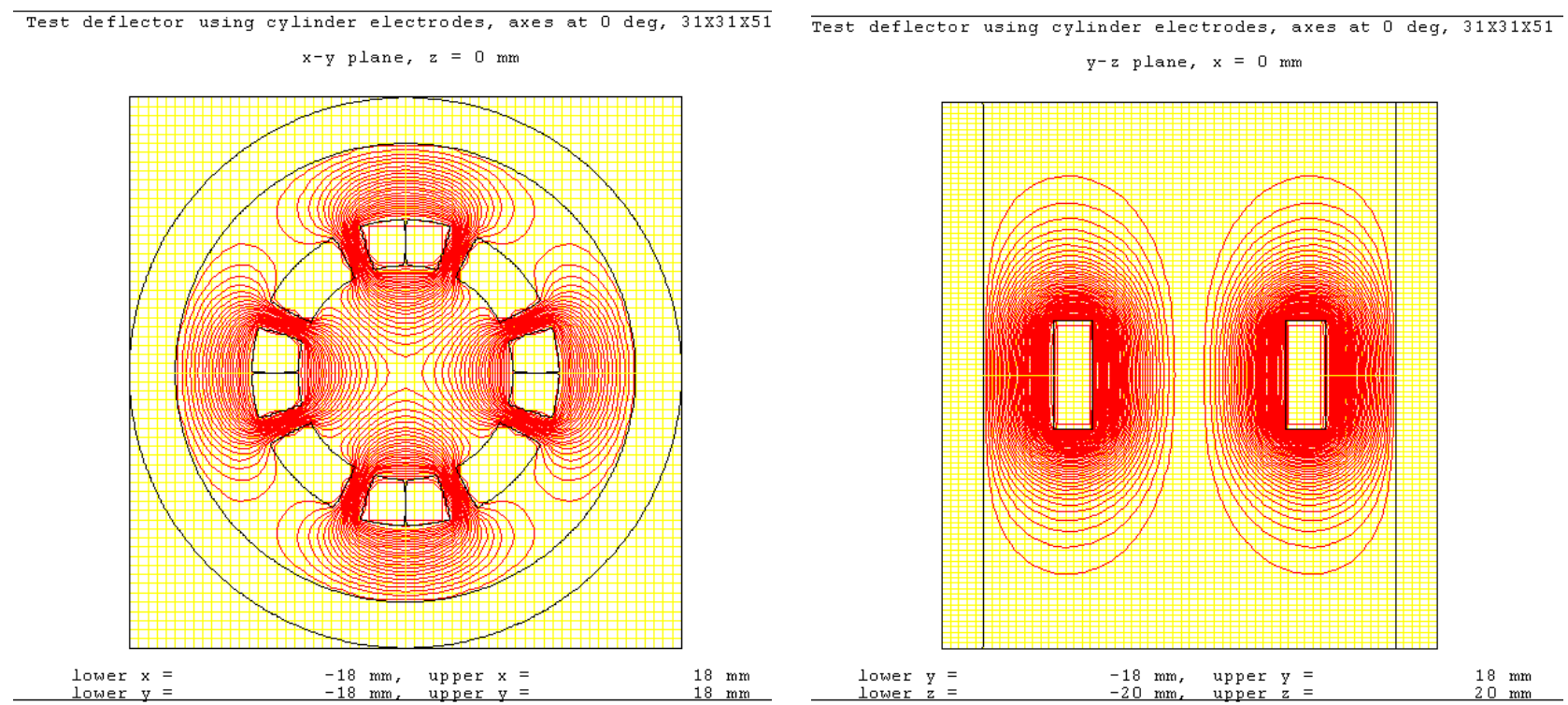

Fig.3 Three-dimension dynamic corrected deflection filed

\section{Realization of Three-dimension Dynamic Correction Field}

The spatial filed of dynamic focus lens at arbitrary point can be obtained by interpolating the fields at mesh points. The fields at mesh points are obtained through the second-order Finite Element Method (FEM) with the relative accuracy of $10^{-15}$. Hermite interpolation is applied to get the field at arbitrary point as it can easily get derivatives with arbitrary order. The spatial filed of dynamic stigmators at arbitrary point will be calculated by the finite difference method through the Mebs software EO-3D. Based on the superposition of electric signals, both the deflection field and the correction one are obtained at the same time as shown in Fig.3. 


\section{Three-dimension Ion Trajectory}

Ion trajectory in three dimensions can be calculated by Newton-Lorentz equations of ions emitting from the source during the sampling time, which is called N-body Monte Carlo method.

\section{Principle of N-body Monte Carlo Method}

The principle of N-body Monte Carlo method is illustrated in Fig.5. Bunches of ions are created near the object plane with random initial positions, directions, kinetic energies and distributions corresponding to the FIB source. The paths of each particle in the bunch of $\mathrm{N}$ particles are computed by solving the Newton-Lorentz equation of motion:

$$
m \mathbf{a}_{i}=q\left(\mathbf{E}_{i}+\mathbf{v}_{i} \times \mathbf{B}_{i}\right) \quad \text { for } \mathrm{i}=1, \ldots, \mathrm{N} .
$$

where $m$ is the mass of ion considering relativity theory, $q$ is the charge, $\mathrm{E}_{\mathrm{i}}$ is the electric field, $\mathrm{B}_{\mathrm{i}}$ is the magnetic flux density, $v_{i}$ is the velocity, $a_{i}$ is the acceleration and the footnote $i$ represents the $i-t h$ particle. The electric field $\mathbf{E}_{\mathrm{i}}$ can be rewritten into $\mathbf{E}_{i}=\mathbf{E}_{L i}+\mathbf{E}_{C i}$ with Coulomb interaction among ions. Where $\mathbf{E}_{\mathrm{Li}}$ is the external electric field and $\mathbf{E}_{\mathrm{Ci}}$ is the Coulomb interaction field. Using Coulomb's inverse square law, the discrete Coulomb repulsion fields $\mathbf{E}_{\mathrm{Ci}}$ can be expressed as:

$$
\mathbf{E}_{C i}=\frac{q}{4 \pi \varepsilon_{0}} \sum_{\substack{i=1 \\(i \neq j)}}^{N} \frac{\mathbf{r}_{i}-\mathbf{r}_{j}}{\left|\mathbf{r}_{i}-\mathbf{r}_{j}\right|^{3}} .
$$

Ion source parameters, the 3D field, and $\mathrm{N}$ should be determined in order to get the ion trajectory.

\section{Realization of N-body Monte Carlo Method}

The parameter of the FIB source is listed in Table 1. Similar to the dynamic correction field, spatial field distribution of lens can be got. As regard to the deflecting field, it has been obtained by calculating the dynamic correction field. $N$ represents the number of ions emitting from the source during the sampling time and it determines the axial length of the bunch $L$.

$$
L=N v q / I \text {. }
$$

Where $I$ is the beam current, $v$ is the speed of ions $\left(\mathrm{Ga}^{+}\right.$is $2.8 \times 10^{5} \mathrm{~m} / \mathrm{s}$ under $\left.30 \mathrm{kV}\right) . \quad N$ can be determined to keep $L$ large enough compared to the physical dimensions of FIB and long enough to eliminate the ending effects. When $L$ is chosen as $10 \mu \mathrm{m}, N$ closed to the source will be 12500 .

Table 1 Original parameters of $0.1 \mu \mathrm{A}$ Ga LMIS

\begin{tabular}{|c|c||}
\hline Class & Ga LMIS \\
\hline Diameter of the virtual source & $50 \mathrm{~nm}$ with Gaussian intensity distribution \\
\hline Energy spread & $5 \mathrm{ev}$, Gaussian distributions \\
\hline Angular current density & $30 \mu \mathrm{A} / \mathrm{sr}$, uniform distribution \\
\hline
\end{tabular}

The parameters of lenses, deflectors, and dynamic correction units are listed in Tables 2 and 3. 3D trajectory is traced by fifth-order Runge-Kutta algorithm with Coulomb interactions. Ion trajectory from $0 \mathrm{~mm}$ and the beam current density distribution at the sample are shown in Fig.4-5 respectively.

\begin{tabular}{|c|c|c|c|c|}
\hline \multirow{2}{*}{ Class } & \multicolumn{2}{|c|}{ Deflection voltage [v] } & \multicolumn{2}{|c|}{ Stigmator voltage [v] } \\
\hline & Deflector 1 & Deflector 2 & Deflector 1 & Deflector 2 \\
\hline $0.3 \mathrm{mmX} 0.3 \mathrm{~mm}$ field & -280.2950 & 359.3526 & -2.1552 & 2.7631 \\
\hline
\end{tabular}
The measured resolution of our FIB system is shown in Fig. 6 under 500pA.

Table 2 Optical parameters of the pre-lens deflectors (Dynamic lens voltage is 5.4273V) 
Table 3 Optical position of the FIB system

\begin{tabular}{|c|c|c|c|c|c|c|}
\hline Class & Source & Lens 1 & Lens 2 & Deflector 1 & Deflector 2 & Sample \\
\hline $\mathrm{z}[\mathrm{mm}]$ & -115 & 90 & 126 & 103 & 118 & 151.5 \\
\hline
\end{tabular}

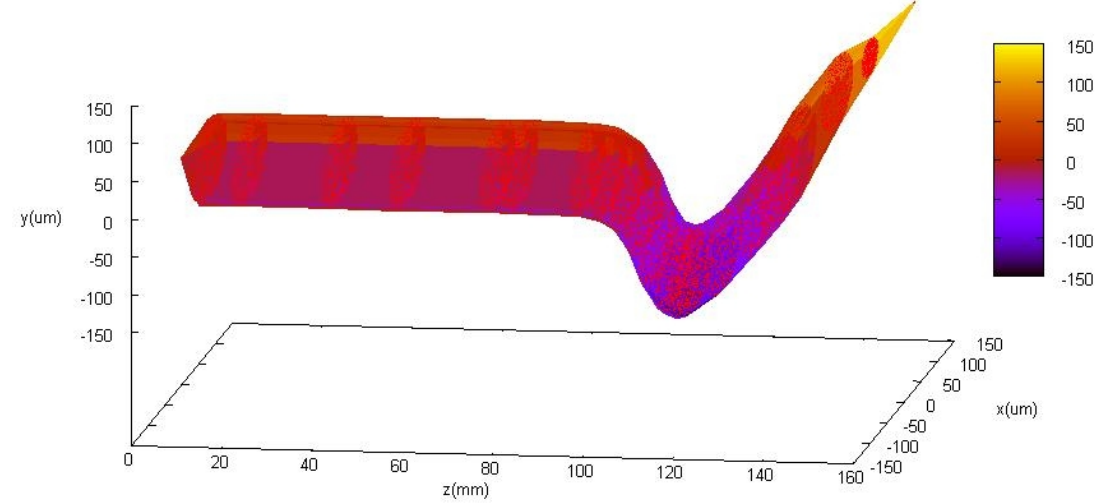

Fig.4 Three-dimension ion trajectory of the FIB system

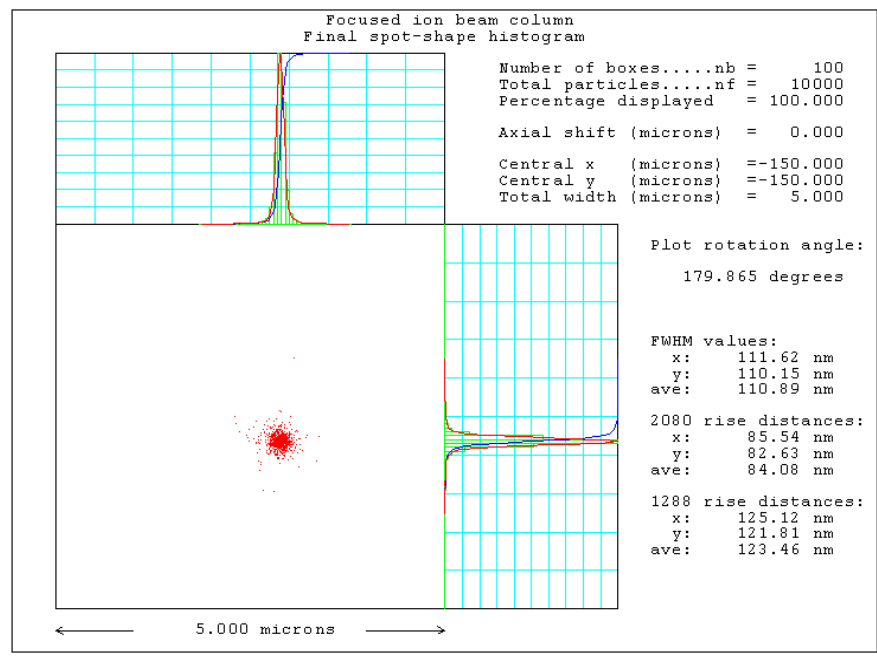

Fig.5 Beam current density distribution at 500pA

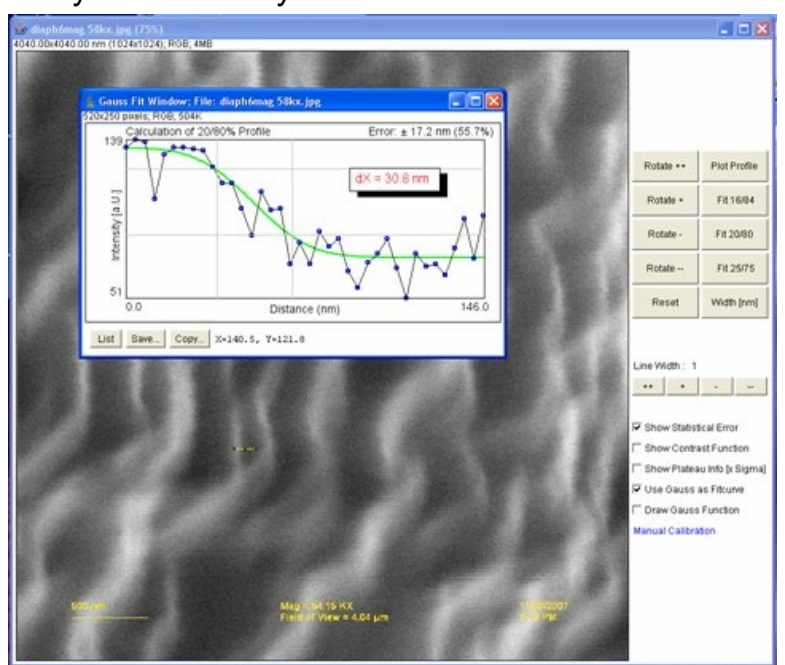

Fig.6 Experimental resolution of our system

\section{Summary}

Controllable incident focused ion beam is realized by simulating Coulomb interactions of a real FIB system with the dynamic corrected deflection field. The beam current density distribution at the sample coincides to the experimental resolution from our Canion $31^{+}$system. 3D ion trajectory from the ion source to the specimen can also lay foundation for the FIB mechanical design. This work is supported by the National Natural Science Foundation of China (Grant No. 11205012) and the Fundamental Research Funds for the Central Universities (Grant No. YWF-15-WLXY-022).

\section{References}

[1] Jon Orloff, Mark Utlant and Lynwood Swanson, High resolution focused ion beams: FIB and its applications, Kluwer Academic/Plenum Publishers, New York, 2003.

[2]Tomáš Radlička, Bohumila Lencová, Ultramicroscopy. 108 (2008) 445-454.

[3] K. Marianowski, T. Ohnweiler, E. Plies, Coulomb interactions in a low-voltage focused ion beam system, Nucl. Instru. and Meth. A 645 (2011) 116-119.

[4]P. Kruit, L.J. Vijgen, Jiang Xinrong, Coulomb interactions in microfocussed electron and ion beams, Nucl. Instru. and Meth. A 363 (1998) 220-224.

[5] J.Bi, P.W.H.de Jager, J.E.Barth and P.Kruit, Influence of Coulomb interactions on current density distribution in a two-lens focused ion beam, Microelectronic Engineering. 41/42 (1998) 249-252. 
[6] Xieqing Zhu, Haoning Liu and Eric Munro, Dynamic correction of aberrations in focusing and deflection system with shaped beams, SPIE 2522: 66-77.

[7]Wenqi Gu, Xiangguo Ma and Wenping Li, Nano-Micro fabrication by focused ion beam, Beijing University of Technology Press, Beijing, 2006. 